\title{
Nos cheveux blanchiront avec nos yeuxde Thomas Vinau :un roman poétique
}

\author{
Fathéya AL-FARARGUY \\ Professeur-adjoint \\ Faculté de pédagogie-Université de Tanta \\ Chercheur associé - centre de recherches \\ sur Zola et le naturalisme de l'Institut \\ des Textes et Manuscrits modernes, \\ UMR 8132 du CNRS-France
}


$-2-$

PDF created with pdfFactory Pro trial version www.pdffactory.com 


\section{Our Hairs will Turn Grey as our Eyes Turn White: poetic novel}

In 'Our Hairs will Turn Grey as our Eyes Turn White', published in 2011 by Alma, the French author Thomas Vinau, born in Tolouz, held a dialogue between nature and life in the location of nature. This novel which is full of poetry was nominated for the European Book Prize 2.

The poetic description of the novel raises a number of questions: What is the poetic novel (poetique) or the novel written in poetry? What are the characteristics of the poetic novel? What are the tools of this visual art of poetic novel? In what way will the reader accept the poetic novel?

To answer these questions, the present study focuses on the works of several critics like: 'The Poetic Story' by Jean Yves Tadié and 'The Descriptive Text: Poetic, Historical and Linguistic by Jean Michel Adam and André Potait John.

The research comprises three points: the issue of the poetic novel and the poetic writing as well as accepting the novel. The former includes two sub-points: the properties of the characters of this form of writing and the time and place poetry, as well as the poetic writing and accepting the novel.

This kind of novel which combines poetry and prose has different names. It is not easy to define the term 'poetique'.

The most important artistic and structural characteristics of the poetic novel are: reducing physical and psychological description of characters for the sake of the place. Here, the location occupies a great area during the several travels of the main character Walther. The location varies from closed to open areas. 
In analyzing the novel the poetic aspect appears saliently in occupying the water areas varying in form the horizontal to the vertical. The contemplation and description of the location reflect the psychological state of the poetic novelist.

Moreover, the internal and external worlds melt through the characters' connection with some objects reflecting feelings. The passing years of age are represented as the rocks eroded by water. At the end of this work, we reach a degree of blending with nature and an image of man composed of water, steam rising or condensed on cold solids, clouds and fog.

The location borders are sometimes not well drawn, and at the same time a kind of unity of the earth and sky.

The time images are rich and characterized by anxiety and the repetition of the images of winter nights. The poetry makes the time eternal and lyrical.

The style of the author is easy and clear, characterized by short sentences, many infinitive verbs and the prevalence of the sub-conscious, approaching closely the automatic writing which is a major characteristic of surialistic writing. The rhetorical phrases show the poetic aspect such as: metaphor, redundancy, juxtaposition, rhythm, homophones, antonyms and development.

At the end of the research we reach the following question: In what way is the poetic novel accepted?

Venault's interest at the end of the novel in the writing process and the necessity of writing, describing the revelation and writing process, the effect of generating vocabulary, the necessity of compromise between man and self and between man and the world remind us of the words of the French author Paul Valerie: "The true meaning of the work of art begins forming in the reader after the navel string tying author and the work is cut." 
This novel has achieved great success in the literature circles and the author has been invited in many forums. This novel has been classified as "among the best works."

The poetic novel was born in the past, then turned to prose style, then back to the poetry style in the twenty first century. This present century witnesses the overlap of forms. The poetry surpasses words and language to concentrate on the poetical viewers and characters.

The richness of the rhetorical style through effective writing, rich description and the reflection of words and images leaves several effects on the reader. The variety of titles and the coordination in the internal structure woven around the times of departure and return, childhood and the hope for the future adds suspense to the plot of the story especially with the wandering of Walter.

Thus, the time and space structure is the corner stone in the poetic novel. The absence of dialogue between the two main characters attracts the reader to follow their correspondences. 


\section{شعورنا ستبيض مع عيونتا \\ للكاتب توماس فينو: رواية شعرية

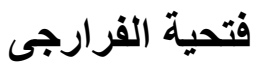 \\ أستاذ (مساعد الفراجد \\ كلية التربية-جامعة طنطا \\ باحث مشارك}

بمعه النصوص والمخطوطات التابع للمركز الوطنى للبحوث العلمية بفرنسا

شعورنا ستبيض مع عيوننا المنشورة عام 2011 بدار ألما، أجرى فيها الكاتب الفرنسـى

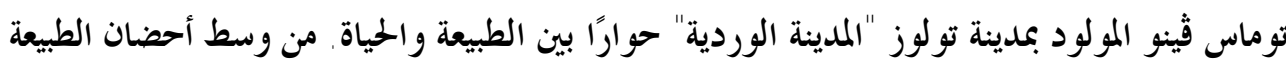

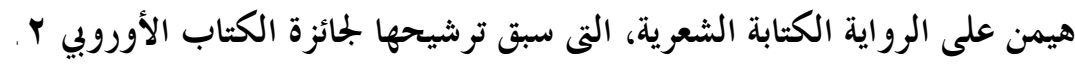

وأمام الوصف الشعرى فى الرواية مجموعة من الأسئلة تطرح نفسها : ما مفهــوم الروايسـة

الشعرية أو الرواية المكتوبة شعرًا ؟ ما خصائص الرواية الشعرية؟ ما أدوات هذا الفن المرئى للرواية

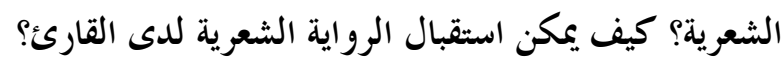

وللإجابة عن هذه الأسئلة ترتكز الدراسة الحالية على أعمال العديد من النقاد نذكر منـــهم

على سبيل المثال: القصة الشعرية لجون إيث تاديه والنص الوصفي: شعرية تاريخية ولغوية نصية لجون

$$
\text { ميثيل آدم وأندريه بوتيت جون. }
$$

وتنقسم خطة البحث إلى ثلاثة نقاط : إشكالية الرواية الشعرية، الخصائص الفنية والتر كيبية

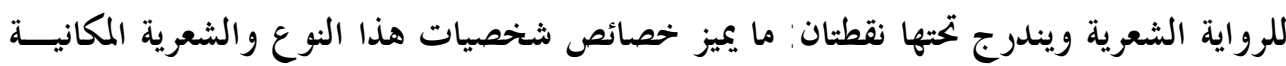

والزمانية والنقطة الأخيرة الكتابة الشعرية واستقبال الرواية.

Poétique تعددت مسميات هذا النوع الروائي الذى يجمع ما بين الشعر والنثر وكلمة كلمة واسعة ليس من السهل تحديد مفهومها.

ومن أهم سمات الخصائص الفنية والتركيبية للرواية الشعرية

$-6-$ 
تضاؤل الوصف الفيزيقي والمعنوي للشخصيات في مقابل أن المكان هو الذى يشغل حيسزًاً

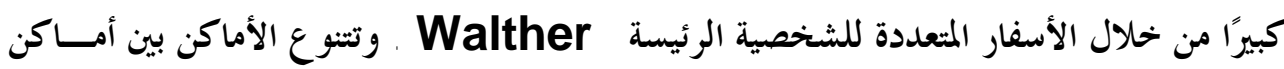

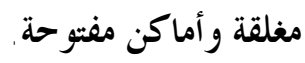

كما يتجلى الجانب الشعري فل تحليل الرواية في شغل المساحات المائية حيزًا كبيرًا وتنوعها في

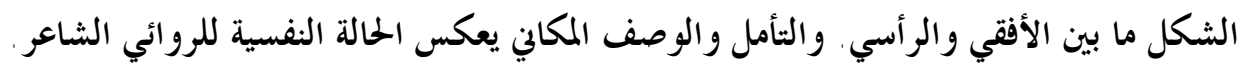
وعلاوة على ذلك يحدث انصهار بين العالم الداخلى والعالم المارجى بارتباط الشخصية بعض

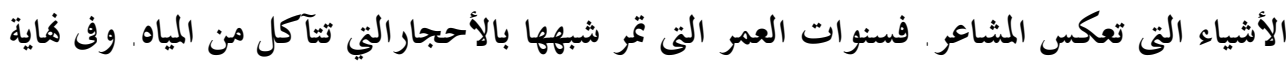

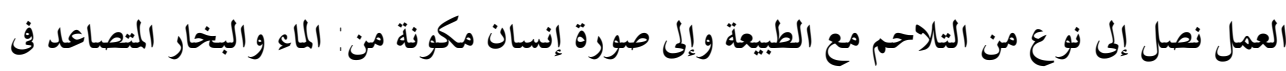

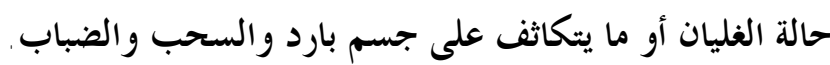
ويتسم الإطار المكاني أحيانًا بنوع من عدم التحديد، وفى ذات الوقت نوع من الشمولية بين

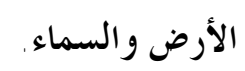

أما عن الصور الزمانية فهى خصبة ويسودها نوع من القلق، ومعاودة الصور الليلية الشتوية.

$$
\text { ويضفى الجانب الشعري على الزمن الحلود والغنائية. }
$$

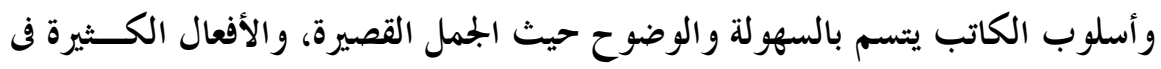

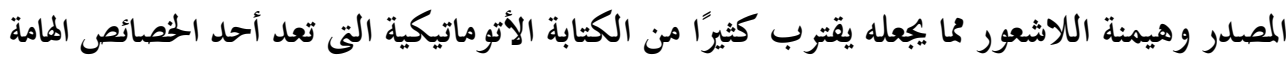

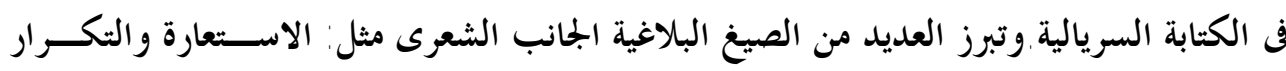

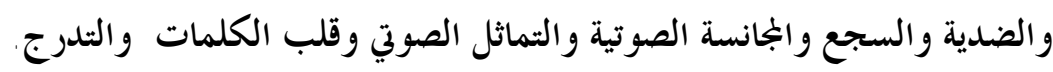

$$
\text { ونصل فل فاية البحث للسؤال الثالي : كيف نستقبل الرواية الشعرية؟ }
$$

إن اهتمام قينو بحديثه في فاية الرواية عن عملية الكتابة، وضرورة الكلام لمن يحب ووصف

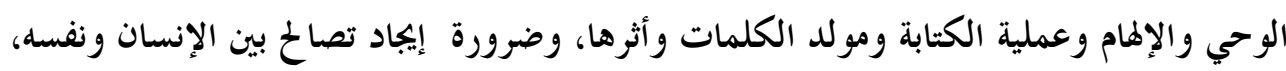

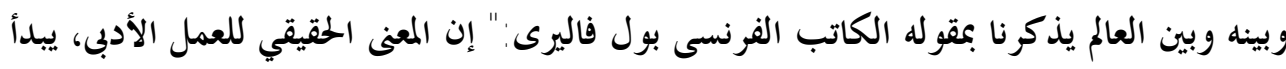
في الثكوين فى نفس القارئ بعد انقطاع الحبل السرى بين العمل ومؤلفها".

$-7-$ 


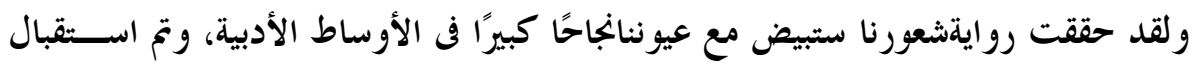
الكاتب في العديد من اللقاءات الأدبية وتم تصنيف الرواية من بين أحسن الأعمال.

هكذا مع الرواية الشعرية يلتقى الماضي حيث ولدت الرواية في قالبها الشعرى ثم تحولهـ

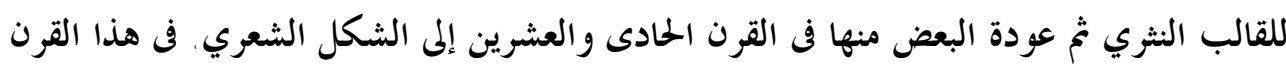

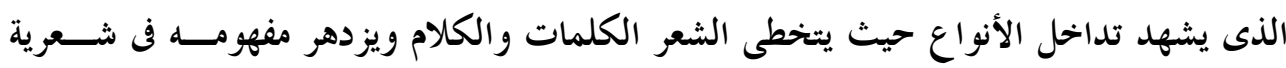

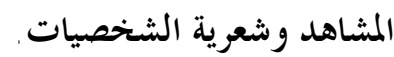

بسبب ثراء الأسلوب البلاغي من حيث الكتابة المؤثرة وثراء الوصف وانعكاس الكلمسات

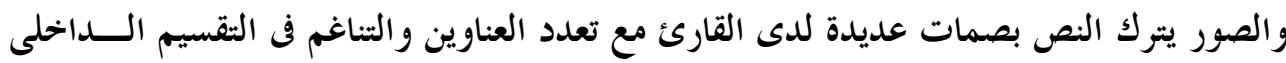

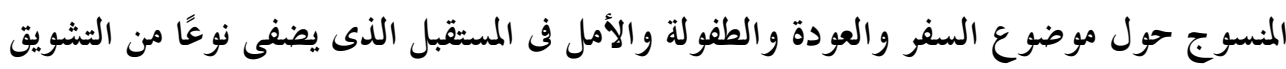

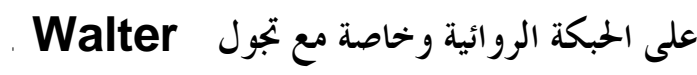
ويعد بذلك التركيب الزماني المكاني هو حجر الزاوية في الرواية الشعرية وغياب الحوار بين الشخصيتين الرئيستين يجذب القارئ أكثر لتبع مر اسلاقمم.

$-8-$ 
Avec son premier roman poétique Nos cheveux blanchiront avec nos yeux ${ }^{(1)}$ publié chez Alma en 201 ' et présélectionné pour le prix du livre européen ${ }^{(2)}$, Thomas Vinauné à Toulouse, la ville rose, et vivant auprès du massif du Luberon entame un dialogue entre la vie et la nature.Au sein de «ce roman-poème composé d'une succession d'instantanés, (il)nous fait partager ses états d'âme dans un jaillissement d'images de haute volée, sans mièvrerie ni lyrisme tonitruant $»^{\left({ }^{3}\right)}$.

Avec le roman poétique s'agit-il d'un retour au premier temps de la naissance du roman ? Est-il une forme hybride? Quelles sont ses caractéristiques structurales et formelles? Comment peut-on recevoir le roman poétique?

\section{I- Problématique du roman poétique}

Le roman français était à l'origine en vers et adaptait des légendes antiques de la littérature latine ${ }^{(4)}$. À la fin du XIXe siècle et au début du XXe siècle, face au déclin du réalisme, et devant la montée du symbolisme, après les deux guerres, Michel

1 Je tiens ici à remercier Monsieur Thomas Vinau qui m'a transmis son roman et d'autres ouvrages, et qui a eu la gentillesse de répondre à mes questions.

2 Midi livre, 23 janvier 2014, dossier de presse envoyé d'Alma.

3 http ://www.lefigaro.fr.site consulté le 18 février 2016.

4 « [Au Moyen Âge]Long récit écrit en roman ou en ancien français, d'abord en vers (notamment en octosyllabes à rimes plates), puis en prose, contant les aventures fabuleuses, galantes ou grotesques de héros mythiques, idéalisés ou caricaturés $»$ http://www.cnrtl.fr/lexicographie/roman 
Raimond constate dans son livre La Crise du roman des lendemains du Naturalisme aux années vingt le fleurissement du roman poétique et la difficulté de sa classification ${ }^{(1)}$. De même, Rachel Grandmangins'interroge sur cet "étrange qualificatif", sur cet " oxymore ", sur cet "objet hybride"du roman en vers. Elle s'appuie sur les écrits de Georg Lukás «Le genre romanesque étant souvent défini comme une dégradation de la noble forme poétique qu'est l'épopée, comme une régression vers le prosaïque, sur le fond comme sur la forme» ${ }^{(2)}$. D'oùle fait que la musicalité, la cadence et la polyphonie accordent à l'œuvre une richesse et lui confèrent une singularité supplémentaire. À la fin deson article deux questions se posent : pourquoi faut-il à tout prix être identifiable comme poète ou romancier? Pourquoi l'œuvre elle-même devrait-elle rentrer dans une typologie ?

Selon Jean-Yves Tadié, le récit poétique en prose né d'une" transition entre le roman et le poème". Du poème, ce récit prend les effets et les moyens d'action et du roman, il tire les techniques de description ${ }^{(3)}$. Pour certains, cette définition est abstraite et plus ou moins arbitraire.

1 Cf. RAIMOND Michel, La Crise du roman des lendemains du naturalisme aux années vingt, Paris, José Corti, 1966, p.194.

2 GRANDMANGIN Rachel, " Le roman en vers », Le magazine littéraire, $\mathrm{n}^{\mathrm{o}}$ 477, Juillet-août 2008, p.18.

3 Cf. TADIÉ Jean-Yves, Le Récit poétique, Paris, Gallimard, 1994, p. 7. 
De toutes les nominations variées de ce roman :"romanpoème","roman en vers", "roman musical", "poésie romancée", "prose poétique", nous (le romancier et moi) préférons roman poétique. Selon Bernard Valette, « la fonction poétique, centrée sur le message lui-même, concerne le style du locuteur, son prosaïsme ou sa poésie, la transparence de sa langue ou ses recherches esthétiques» ${ }^{(1)}$. Pour $\mathrm{T}$. Vinau qui a publié une quinzaine de recueils, cette pratique poétique lui a redonné l'occasion pour revenir au roman en $2010^{(2)}$. La poésie brise toutes les frontières, mélange raison et imagination. Héritier de tout ce développment poétique surréaliste, T. Vinauinvente un roman avec une poésie mouvante.

\section{II- Les caractéristiques formelles et structurales du roman poétique}

\section{1- Les caractéristiques formelles}

Avec sa couverture grise claire et les avocettes bleues, précédant la dédicace à l'un de ses fils, Gaspard, on lit l'indication :ouvrage publié sur la recommandation de JeanBaptiste Gendarme, le rédacteur en chef de la revue Décapage,qui, faisant partie du comité de sélection d'Alma et a

1 VALETTE Bernard, Le Roman: initiation aux méthodes et aux techniques d'analyse littéraire, Paris, Armand Colin, 2011, p. 61.

2 Cf. Thomas Vinau chez Obliques, Cf. L'Yonne Républicaine, 24 novembre 2014, dossier de presse envoyé d'Alma 
proposé le manuscrit du romancier. T. Vinau considère la couverture du livre comme un visage et le titre son regard ${ }^{(1)}$.Cette définition personnifiée donne un effet poétique comme s'il s'adressait à une personne. Une personnificationmise en relief par la description du moment de la naissance titrede l'œuvre comme s'il marchait sur les pieds ${ }^{(2)}$. C'est ainsi qu'avec Nos cheveux blanchiront avec nos yeux, le lecteur pourra s'interroger sur le choix de l'adjectif possessif " nos", tout comme il s'interrogera sans doute sur l'image des cheveux qui blanchissent avec les yeux.Cette image comparatived'ailleurs répétée dans certains titres des chapitres de l'œuvre : "Des milliers de gris", "La crête blanche du temps " démontre l'art dubon écrivain qui peut choisir des images neuves ${ }^{(3)}$.Toutefois, certains voient que les yeux ne vieillissent point, c'est-à-dire leur aspect ne se modifie pas au fil des années, d'où la jeunesse du regard de certains vieillards. C'est ainsi que le titre devient une invitation à la lecture par la poésie discrète, celle des oiseaux de passage, des gens doux qui écoutent Bob Marley en voiture ${ }^{(4)}$.

1 Cf.http://etc-iste.blogspot.com.eg/sité consulté le 19 février 2106

2 Cf. Ibid.

3 Cf.SAYED Fathi, La Syntaxe et l'effectivité, Le Caire, Les amis du livre, 2000, p.30.

4 Cf. PlOUGASTEL Yanne, Le Monde Magazine, 10 sept 2011, dossier de presse envoyé d'Alma. 
L'alternance entre versification et prosese constitue autour d'une forme poétique présentée en " drapeau" et en une "succession de blocs introduits par un titre "(1) et le fond d'un roman composé de deux parties avec des titres alternatifs contradictoires et complémentaires: " Le dehors du dedans" et "Le dedans du dehors". Ces titres sontsuivispar deux exergues : «quand on aime il faut partir » de Blaise Cendrars et« j'ai l'obstination farouche d'être doux»de Victor Hugo. Les deux parties mènent ainsi à deux modes de dénouement : une coupure avec le départ de Walther qui représente l'acmé de l'action et une conversion avec son retour du voyage.

Chaque partie estannoncée par des titres en caractères gras : soixante-cinq titres pour la première et soixante-treize pour la deuxième. Le roman est présenté sous forme de tableaux d'images prises sur le vif, de scènes fragmentées et d'instantanés: «chaque fois une brassée de phrases mises en page comme des vers libres, avec un titre $»^{(2)}$.Ces titres représentent un tableau complet :le goûtdu biscuit, du lait et du sel; le son : la sonate du lampadaire; les sentiments : trois tonnes et une larme. En outre, certains titres soulignent le côté narratif surtout par la répétition

1 Ibid.

2 Libération, 22 septembre 2011, dossier de presse envoyé d'Alma.

$-13-$ 
d'une histoire de"crocs et d'orange", de"chaussure", de "ligne et de pelle".

Le romancier termine la première partie avec l'annonce de la deuxième via les titres suivants : "Où il est question de savoir où l'on se sent le mieux" et "Naître dans les décombres ". Avec cet autre titre : "La boucle", T. Vinau présente un épisode crucial du roman parce que son personnage principal, Walther, change. Enfin, le roman se termine par un "Autoportrait". T.Vinau, romancier poète, peintre, ce Pertuisien toulousain «s'approche d'Edward Hopper et colle son oil de connaisseur au trou de la serrure pour focaliser une foule de détails caractéristiques de celui qui croyait, avant tout, au bien fait de l'improvisation soudaine ${ }^{(1)}$.

Les détails caractéristiques s'avèrent structuralement par la spécificité des personnages et le cadre poétique spatial et temporel.

\section{2-Les caractéristiques structurales}

\section{A-La spécificité des personnages du roman poétique}

Sous le titre : " La boucle", le romancier note le prénom des personnages verticalement en commençant et en terminant par

1 MITCHEL Anne-Marie, "Réalisme d'ambiance », La Marseillaise, 7octobre 2012, dossier de presse envoyé d'Alma. 
Sally. Les autres sont Kavlar, Lenka, Éric, Thala, Pec, Elisa. Mais il ne cite pas Walther. Comme les personnages surréalistes de faux roman, les personnages de la présente étude sont mis sur scène par «un petit nombre de caractéristiques physiques et morales» ${ }^{(1)}$.Pour Daniel Berland, ce texte est fort parce qu'il oscille entre carnet de route, poésie et introspection. Il accorde à l'auteur et l'éditeur un grand success ${ }^{(2)}$.

Ainsi le lecteur peut-il cerner leur psychologie grâce à leur incarnation dans l'action. Dans Nos cheveux blanchiront avec nos yeux, l'action est tissée de diverses manières : la présentation de l'idée principale du départ, un coup de téléphone entre Sally et Walther pour lui annoncer son besoin de voyager, un dialogue au port entre le capitaine et les demandeurs d'emploi.

L'idée de départannoncée dès l'incipit du roman est comparée à «un petit feu de bois placé au centre de son cerveau» ${ }^{\left({ }^{3}\right)}$. Cette comparaison entre l'idée et le feu de bois évoque le tableau de Walther brûlé par ses idées. A tel point qu'il

\footnotetext{
1 BRETON André, Manifeste $d u$ surréalisme 1924, https://poesiemuziketc.wordpress.com/2013/03/29/andre-

breton-manifeste-du-surrealisme/ consulté le 6 mai 2016 consulté le 6 mai 2016.

2 Cf.BERLAND Daniel, Fragments d'un immobile voyage, Page, octobre 2011, dossier de presse envoyé d'Alma.

3 VINAU Thomas, Nos cheveux blanchiront avec nos yeux, Paris, Alma, 2011, p.13. Dorénavant nous utiliserons l'abréviation N.C.B.A.N.Y pour Nos cheveux blanchiront avec nos yeux.
} 
domine son existence. Le voyageur Walther, menacé de suffocation, se promène en solitaire dans un road-movie, des Flandres à l'Espagne. Comme Pierre de Reverdy, ce personnage est tenaillé et déchiré entre le rêve et la réalité( ${ }^{(1)}$.

Cette idée principale incarne la sédentarité et l'errance physique comme morale. Elles sont mises en lumière par le romancier qui déclare: «Je suis l'architecte de la fuite, mon labyrinthe est en ciment $\|^{(2)}$. Ce "militant du minuscule"(3) construit le rêve de son protagoniste Waltheren vagabondant d'un pays à l'autre, d'un voyage intérieur à un voyage extérieur en anticipant sa chute par son état dépressif4.

Pendant ses voyages, Walther garde en lui, la présence de Sally grâce à leur correspondance décrite comme: « des tunnels entre l'espace et le temps» (N.C.B.A.N.Y, p.54), comme : «un jour blanc à écrire des lettres qui n'en finissent pas », « un jour où il est possible de dire aux gens qu'on les aime » (N.C.B.A.N.Y, p.67).Cette correspondancepermet au lecteur captivé, de découvrir leur personnalité. Cette écriture à distance

1 Cf. ASTRE Marie-Louise et COLMEZ Françoise, Poésie française : anthologie critique, Paris, Bordas, 1982, p. 379.

2 Décapage, printemps-été 13, dossier de presse envoyé d'Alma.

3 www. vogue.fr

4 BERLAND Daniel, Fragments d'un immobile voyage, Page, octobre 2011, dossier de presse envoyé d'Alma 
montre l'originalité du romancier et son pouvoir faire entendre: «le cœur battant d'une présence $»^{(1)}$.

Cette relation, faite de présence et d'absence entre les deux personnages est présentée dans un poème. Pour donner l'effet sonore de la lecture rythmique entamée par Linka, le romancierpoète y utilise les barres obliques :

«Tu vois / il y a l'histoire de ce loup/ qui se laisse

Apprivoiser/civiliser/Et puis il y a l'histoire de ce chien / qui retourne à l'état sauvage /qui se libère dans la forêt/ et moi je préfère / laseconde histoire/ même si ma vie /même si ma vie ressemble de plus en plus à la première /...» (N.C.B.A.N.Y, p.23).

Walther cite le lieu d'où il écrit de l'autre côté du pont. Il met l'accent sur l'influence qu'exerce sur lui cette écriturela comparant à des "Pansements".L'effet de répétition dans "même si ma vie" souligne son hésitation entre les deux histoires du loup et du chien.

D'autre part, la présence du romanesque poétique est accentuée par la domination des rêves. Walther obsédé par son rêve d'un escalier de pierre qui descend vers la mer à Portbou. Ce

1 DELABROCHE Philippe, Lire, septembre 2011, dossier de presse envoyé d'Alma.

$-17-$ 
rêve se prolonge par une recherche de l'horizon: «Entre les mailles de la glycine glacée, un bout de lune pris au filet»(N.C.B.A.N.Y, p.69).

Le lecteur peut arriver à une découverte, grâce au « lyrisme de l'expression», à «la chaleur de l'inspiration», à«la qualité de l'atmosphère $»^{(1)}$ et à autant d'éléments présentant bien d'autres aspects concrets et essentiels qui rappellent la mémoire de l'enfance dans un lieu plus ou moins clos et coupé de la réalité.

Mais pourquoi le départ dans la première partie et le retour dans la seconde? Ce retour à la famille est un des sujets primordiaux de la plupart des premiers romans ${ }^{(2)}$. Sur le plan narratif, Walther revient pour «devenir le narrateur de sa propre vie $\gg 3$.

D'après l'analyse des prénoms de Jean-Marc de Foville, Walther est la forme germanique de Gautier qui est un combattant qui épuise son entourage par «sa mobilité ", " son enthousiasme sans cesse renaissant », " sa curiosité d'esprit »4. Le romancier l'assimile à V. Hugo en citant les vers suivants : «

1 DELABROCHE Philippe, Lire, septembre 2011, dossier de presse envoyé d'Alma, p.195.

2 Cf. Livres de France, septembre 2011, dossier de presse envoyé d'Alma.

3 DELABROCHE Philippe, Libération, 22sept 2011.

4 DE FOVILLE Jean-Marc, Les Prénoms de vos enfants : origine, histoire, signification, Paris, Hachette,1995, pp. 303, 637. 
J'étais le vieux rôdeur sauvage de la mer/ Une espèce de spectre au bord du gouffre amer »(N.C.B.A.N.Y, p.72). C'est à travers le regard de Walther que le romancier a rédigé ces belles pages avectoutes ces phrases courtes, le lecteur peut trouver« tous les matins du monde $»^{(1)}$.

Quant au deuxième personnage : Sally, prénom dérivé de Sarah, on l'associe à lapersévérance, à lafécondité par son amour des enfants, à l'audace intellectuelle ${ }^{(2)}$. Avec l'enfant de Sally, le roman poétique se caractérise par l'existence d'un personnage à naître dont l'absence de prénom donne un effet de généralisation : « Il est Dieu, il crée le monde qu'il perçoit, ne dissocie pas l'un de l'autre »(N.C.B.A.N.Y, p.60). Cette future existence donne une sorte d'ouverture vers l'espoir. Walther montre d'une manière poétique et sensible comment il devient père en s'interrogeant comme peut le faire tout un chacun dans cette situation :

Où est-il question de savoir où l'on se sent le mieux ? Qu'est-ce que j'en fais-moi de tout ça?

1 PLOUGASTEL Yanne, Le Monde Magazine, 10 septembre 2011, dossier de presse envoyé d'Alma.

2 Cf. DE FOVILLE Jean-Marc, op.cit., p.573. 


\section{B-Poétique spatiale et temporelle}

Devant l'effacement des personnages, l'espace occupe un rôle primordial et les décors «urbains ou naturels » ont une place privilégiée. Pour Tadié : «ces paysages ne sont, eux aussi, que des êtres du langage $\gg{ }^{(1)}$. La représentation paysagère transmet un langage très éloquent par l'écho de son silence, par le reflet de ses couleurs et ses dimensions. Une langue sans nationalité et sans arrière fond culturel ou social ou politique. En un mot, c'est un langage naturel.

Entre des lieux ouverts qui dominent la scène romanesque : (au port, sur le bateau, Amsterdam, L'Achab et la Terre Neuve, la Hollande, la mer du Nord, l'île de Spitzberg ), et des lieux fermés :( dans une chambre d'hôtel ), on assiste à une biographie réinventée à partir de miettes poétiques. Rappelons à cet égard que Christiane Lahaiedésigne par «le mouvement imagiste de la poésie moderne ». D'après ce mouvement le texte littéraire est construit plutôt d'après une logique spatiale ${ }^{(2)}$. D'où $T$. Vinau préfère et se passionne pour une chambre $\mathrm{d}^{\prime}$ hôtel ${ }^{(3)}$ vide ou un réverbère ${ }^{(4)}$.

1 TADIÉ Jean-Yves, op.cit., p.9.

2 Cf. LAHAIE Christiane, Entre géographie et littérature; question de la mimèsis, Cahier de géographie du Québec, vol.52, n147, 2008, pp.439-451.

3 LORET Éric et BOURMEAU Sylvain, Ces images correspondent à mon travail, Libération, 6/7 octobre 2012, dossier de presse envoyé d'Alma.

4 Cf. VINAU Thomas, Bric à BracHopperien, Paris, Alma, 2012, p. 14. Edward Hopper (1882-1967)Anthony Dufraisse écrit : « Ce recueil aux accents 
La richesse poétique provient de l'entrecroisement des images descriptives. L'espace est structuré essentiellement autour de Walther mis en scène dans un premier tableau présenté et annoncé par l'adjectif possessif à la troisième personne : «Sa façon de disparaître à travers ses yeux lorsqu'elle regarde la fumée des cheminées d'usines se mélanger au gris de l'océan»(N.C.B.A.N.Y, p.13). Cette disparition assimilée à la fumée des cheminées et accompagnée de la musicalité des mots se termine par le son [e]. Quelques pages plus tard, par une sorte dechiasme qui donne un effet de miroir par des mots et des images, le lecteur devient plus proche de la réaction des deux personnages.Sally s'adresse à Walther en avouant : « j'observe la fumée des cheminées se mélanger au ciel, je repense à ta façon de regarder à travers la vitre de la chambre » (N.C.B.A.N.Y, p.27).

Selon les théoriciens, la description peut avoir trois fonctions majeures : mathésique (diffusion du savoir), mimésique (construction d'une représentation) et sémiotique (régulation du

autobiographiques ne manquera pas de charme pour tous ceux pour qui l'écriture a partie liée avec le transitoire, le passager, l'écume. Intérieur ou extérieur, le monde de T. Vinau se donne sans artifices ».DUFRAISSE Anthony, "In Vinauveritas ", Le Matricule des anges, fev 2014, dossier de presse envoyé d'Alma. 
sens) ${ }^{(1)}$. Pour Nos cheveux blanchiront avec nos yeux, c'est la fonction mimésiquequi domine dont le rôle «unique ou principale, est de mettre en place le cadre de l'histoire, l'espacetemps dans lequel les acteurs interagissent » dont les deux enjeux majeurs sont :

1- Construire l'espace-temps du récit, son "chronotope", son "autoréférence, sa "socialité",

2- Présenter les acteurs de l'histoire ${ }^{(2)}$.

La poéticité spatiale de Nos cheveux blanchiront avec nos yeux s'interprète par des paysages aquatiques sous deux formes : horizontale avec l'étang ; la rivière et verticale avecla pluie et la neige. A travers l'image métaphorique de l'Europe, le héros se voit comme un poisson maigre. Puis il passe à une image terrestre :

\section{«l'Europe est un immense filet glacé Et moi je suis un de ces poissons trop maigres »(N.C.B.A.N.Y, p.31)}

Du délire du rêve au délire de l'alcool, il imagine une ville dont il accentue l'importance et en dresse un tableau avec le reflet des couleurs des plantes sur les fenêtres. Dans cette ville, il s'imagine comme une page blanche et personnifie ses sensations, les odeurs et les images :

1 ADAM Jean-Michel et PETITJEAN André, Le Texte descriptif: poétique historique et linguistique textuelle, Paris, Armand Colin, 2006, pp.33, 36.

2 Cf.Ibid., pp.33, 36. 
«Dans les ruelles, les murs sont délabrés. La chaux très colorée donne du cachet aux encadrements de Fenêtres»(N.C.B.A.N.Y, p.45).

Ce rêve crée un espace intérieur et la description peut avoir une autre fonction signification contextuelle et référentielle en décrivant moins le mondevisible ${ }^{(1)}$. Là, il s'agit aussi du troisième type de la description dite expressive "selon l'âme du poète"(2).

Le romancier imagine des nuages qui rient et voit le ciel comme un être vivant avec des dents «Le rire gras des nuages se moque de tout le monde. Le ciel a les dents blanches, je le vois ricaner».La personnification du ciel et des nuages par ces images métaphoriques incarne l'importance accordée aux éléments de la nature plus qu'à l'existence humaine. L'espace intérieur se modifie avec la fuite de l'obscurité et la naissance d'un nouveau jour. La scène du monde lavé par la lumière rend l'image très évocatrice. La pluie accompagne Walther qui se lève avant la montée du jour et annonce l'arrivée de la maternité.Devant cette pluie bien droite et verticale, la vision de soi et du monde s'enchaîne :

«Les yeux en face des trous. Elle coupe le paysage en tranches.

1 Cf. VALETTE Bernard, Le Roman : initiation aux méthodes et aux techniques d'analyse littéraire,Paris, Armand Colin, 2011, p. 34.

2 ADAM Jean-Michel et PETITJEAN André, op.cit., p.8. 


\section{(...) J'ai l'impression d'être de plus en plus loin de ce que je vois. De plus \\ en plus loin à l'intérieur de moi» (N.C.B.A.N.Y, p.59).}

La description est expressive parce qu'elle se présente comme le dépositaire d'un point de vue, qu'il soit celui de l'auteur ou celui du personnage. Ce qui se manifeste textuellement par la présence d'isotopies euphoriques ou dysphoriques, selon "l'état d'âme" du descripteur et par une condensation de marqueurs de subjectivité( ${ }^{(1)}$. La force évoquée par les nuages qui "se dressent", "se musclent", "s'étendent", par les giboulées qui «giflent le monde d'une impertinente cadence », par le vent qui «frappe d'un côté, de l'autre », par la pluie qui «tombe en bourrasques, s'enroule, retombe, file dans tous les sens en flèches folles $\gg{ }^{(2)}$.

$\mathrm{La}$ personnification des nuages rend la description expressive accentuée par la pluie qui "secoue", "décoiffe"(3) . La médiation spatiale de ses atmosphères "urbaines", "nocturnes", "souvent pluvieuses" reflète l'état d'âme du romancier-poète par ses images "plus organiques", "plus musicales" ${ }^{(4)}$.Le mouvement de la pluie domine l'image aquatique avec une rapidité de rythme qui transmet une sorte de possession et une globalité.

\footnotetext{
ADAM Jean-Michel et PETITJEAN André, op.cit., p.8.

2 VINAU Thomas, La Part des nuages, Paris, Alma, 2014, p. 61.

3 Ibid., p. 62.

4 Cf. V. R., Entre chien et loup, Livres Hebdo, n 869, vendredi 10 juin 2011 dossier de presse envoyé d'Alma
} 
Dans son deuxième roman La Part des nuages, T. Vinau montre l'effet de cette pluie tombante qui le rassure ${ }^{(1)}$. À travers l'image accordée au ciel, il évoque la protection; il assimile le ciel à un stylo qui se remplit d'encre et le personnifie : «Électrique. C'est plutôt beau quand l'horizon s'énerve. Que les pistes se brouillent $»^{(2)}$. D'où le besoin pour un abri comparé à une couverture. De la pluie à la neige, de l'image de l'eau fluide à l'image de l'eau glacée puis de celle de l'aube. Face à cette scène froide couverte de nuage, d'autres tableaux se dessinent avec le rayonnement du soleil : «Les pierres vertes des murets ont les cheveux qui poussent»(N.C.B.A.N.Y, p.77).La diaphore ou répétition d'un même mot déjà employé donne une nouvelle nuance de sens ${ }^{(3)}$ : « Le soleil éblouit. Un drôle d'oiseau pousse son drôle de cri. J'explore les éboulis. Ton rire dévale la pente et vient se fracasser dans la lumière»(N.C.B.A.N.Y, p.91).

La fusion de deux mondes : extérieur et intérieur transmet des sentiments par l'attachement à certains objets. Walther ressemble à un galet qui reflète ses sentiments, puis s'adresse à sa bien-aimée à l'aide du pronom d'insistance: toi dont son absence est une source de souffrance. Après ces séries de scènes

1 Cf. VINAU Thomas, La Part des nuages, Paris, Alma, 2014, p. 22.

2 Ibid., p. 61.

3 BÉNAC Henri et RÉAUTÉ Brigitte, Vocabulaire des études littéraires,Paris, Hachette, 1993, p.63. 
aquatiques, le roman poétique transmetl'image de l'être humain composé d'eau, de buée, de vapeur, de nuages, de brumes qui s'évaporent. Le romancier donne à la fin une sentence : «Vivre consisterait ainsi à s'vaporiser. L'âme serait un reflet sculpté dans le miroir ». Le roman se termine par un dernier titre : "Ça suffirait". L'homme a une existence rapide et éphémère, mais il peut laisser ses traces après son départ.

Quelques fois, le cadre spatial se caractérise par l'indécision : une île sans route, l'horizon en ciment, la globalité créée par l'image céleste et terrestre. Cette indécision spatiale amplifie l'effet poétique du roman par la valorisation du paysage dans n'importe quel lieu. Le romancier ne s'enferme pas dans un endroit précis; ce qui donne l'effet d'une vision et d'une existence humaine universelles. Cette indécision peut également démontrer une sorte d'effacement des frontières comme dans la plupart des pays européens. Une sorte d'entretien avec des terrains inconnus et des identités perdus.

Quant aux images temporelles, elles sont fertiles. T.Vinau justifie sa profession de poète dans sa lettre à Jules Mougin : «Je serai poète pour me manger en temps de crise $\rangle^{\left({ }^{1}\right)}$. Cette phrase accorde probablement une réponse à la question suivante :

1 Décapage, hiver 13-printemps-été 14, dossier de presse envoyé d'Alma. 
pourquoi Vinau a-t-il choisi cette forme poétique plutôt qu'une autre ? Pour des considérations d'ordre culturel, avec l'écriture poétique, les mots comptent et expriment plus profondément les sentiments. Par ailleurs, Vinau met les mots dans un tissage d'une histoire pour entrelacer tous ses fragments poétiques dans un seul fil. Comme un photographe qui prend une succession des clichés les plus divers pour les mettre dans un seul album.

Vinau a écrit un roman court pour se mettre au niveau d'un lecteur contemporain. Le rythme accéléré du XXIe siècle, pousse les nouvelles générations à s'intéresser plutôt à des formes plus courtes. La plus grande majorité des lecteurs n'a probablement plus la patience de lire des romans longs. Toutefois il nous semble que l'écriture poétique (même si elle est plus rapide à lire) est moins accessible que l'écriture en prose.

Il écrit ce roman à l'âge de vingt-neuf ans, toutefois il ressent précocement la vieillesse. L'image temporelle est dominée par un sentiment d'inquiétude accentuée par l'anaphore "peur de" : "de grandir", "de devenir comme tout le monde", "d'accepter cette drôle de farce ", " de la médiocrité ","de ce qu'il allait devenir ", "de ce que j’étais devenu ","Maintenant j'ai peur de ce que je ne deviens pas ". Après cette répétition douze fois, après l'évocation de son angoisse du passé, 
du présent et du futur, le romancier termine ce tableau par « Je n'ai plus peur. J'en ai juste marre $»^{\left({ }^{1}\right)}$. Nous constatons ici, la polyptote du verbe devenir.

Le temps des verbes dont le futur, employé dans le titre, annonce la destinée du pronom collectif "nous". C'est le sort fatal de tout un chacun. Le romancier met devant les yeux du lecteur, la progression de l'image de la vieillesse. Il termine ce tableau par la séparation de l'être humain au monde à travers l'éloignement des choses et sa disparition graduelle : «On finit seul, en haut de la tour à regarder les choses bouger loin de nous »(N.C.B.A.N.Y, p.60).

Les verbes placés au début transmettent un effet d'insistance et de mise en valeur dont l'emploi de l'infinitif renforce les autres verbes grâce au témoignage de celui qui parle : «Prendre la vie comme elle vient. Vite. En passant. Entre deux portes et deux soupirs. Entre deux coups de vent. Prendre ce qu'elle nous donne. Appeler quelqu'un qui compte. Sentir l'herbe coupée »(N.C.B.A.N.Y, pp.95-96).La présente image poétique traduit le rythme accéléré de la vie dès le cri de la naissance au soupir de l'agonie. C'est pourquoi, il pense à ses vingt ans et insiste à l'aide du présentatif "c'est" sur le fait d'accepter ou de partir.

1 VINAU Thomas, La Part des nuages, Paris, Alma, 2014, p. 95. 
Les années de la vie qui passent sont assimilées aux pierres usées par l'eau : l'image du monde qui nous grignote. Cette sorte de contradiction indique au lecteur la réaction du personnage principal vis à vis du temps. La scène de l'attente de la disparition de l'obscurité et de l'apparition du jour est auréolée par des éclats d'argent et de verre : " nos nuits ont brûlé dans des éclats d'argent et de verre pilé »(N.C.B.A.N.Y, p.101).

Les phrases courtes sans verbes ou les verbes à l'infinitif ou les phrases en suspens donnent l'effet d'un style énumératif «qui rappelle les sonates de Schubert ${ }^{(1)}$. Avec de rythme accéléré et successif, avec cette écriture plutôt automatique ou cette dictée de l'inconscient émerge un monde magique ${ }^{(2)}$. Pour Philippe Zamari, ce roman est écrit avec un« style dépouillé mais d'une grande recherche formelle, aux fulgurances étonnantes. Un ovni littéraire, certes, mais à observer de près $\rangle^{\left({ }^{(3)}\right.}$.

L'affectivité du côté poétique est traduite parla recrudescence d'images nocturnes hivernales et matinales : "La route de la nuit ", "Au milieu de la nuit ", "Avant l'hiver/Avant la nuit", "Les matins qui m'éloignent de toi sont des nuits ", "Un

1 BARLAND J-R., Thomas Vinau : homme cherchant refuge, La provence, 19 octobre 2014, dossier de presse envoyé d'Alma.

2 BOUQUET Dominique, Le Surréalisme en France et en Europe, Collection les guides Pocket Classique, Paris, Pocket, 2003, p.58.

3 ZAMARI Philippe, La BD en flagrant délire de Desproges-mania, Nice Matin, 27 novembre 2011, dossier de presse envoyé d'Alma. 
éclat minuscule ", "Le soleil de minuit", cet oxymore intensifie l'existence et l'émergence de l'espoir. Dans d'autres titres: l'accent est aussi mis sur le mois d'avril: "La colline d'avril", "Les soirs d'avril se partagent". Il nous donne l'image d'un jour qui naît.

Les images matinales contredisent l'image nocturne hivernale. Il donne au matin l'image d'un corps, voire l'odeur qui devient quelque chose qu'on va écouter : «Je colle mon oreille à ta peau. J'écoute bouillir ton corps. J'écoute ton odeur quand la lumière s'infiltre au sommet des volets fermés. Mes yeux ne voient pas droit. Ils mélangent le ciel et la terre. La pluie et la rosée »(N.C.B.A.N.Y, p.102).

La cohérence du cadre spatio-temporel met en relief l'action et rend le temps éternel et lyrique. Ce lyrisme émerge d'un effet d'harmonie produit par l'allitération qui est bien présent : retour des mêmes sons consonantiques de l'occlusive [p] et l'assonance : retour des mêmes sons vocaliques en a/an «Un pansement. Une pensée. Une persistance. Une attention. Une allumette » (N.C.B.A.N.Y, p.68). 


\section{III- L'écriture poétique et l'invitation à sa réception}

Pour T. Vinau, l'écriture est une « discipline vitale quotidienne» et le poème est une « gymnastique $»^{\left({ }^{1}\right)}$.

À la question : comment peut-on recevoirle roman poétique ?, ilrépond en avançant le fait d'écrire d'après une tendance à aimer ou ne pas aimer parler. Il annonce qu'il est plus proche de la deuxième catégorie. Pour cette raison, il cite Marguerite Duras : «Écrire c'est se taire »dans la dernière page, intitulée Autoportrait. Il se définit et s'assimile aussi à " une fenêtre", à "une présence derrière un rideau diaphane"(N.C.B.A.N.Y, p.95). Mais par la comparaison et l'image de l'esprit qui donnent plus de profondeur à cet acte, cette invitation et cette incitation à l'écriture devient très rhétorique : "L'esprit comme du vent dans une longue plaine. Une langue pleine. Les mots qui fouettent l'herbe, qui disparaissent en bourrasque». Le fouettement des mots estrenforcé par l'homophonie des homonymes, la paronomase : une longue plaine, une langue pleine.

Cette langue pleine émerge d'une lucidité, d'un murmure qui a pour effet de «Retourner d'où l'on vient. Droit. Les yeux

1 Livres hebdo, 10 janvier 2014, dossier de presse envoyé d'Alma. 
bien en face du grand trou de nos vies ». Comme chacun a sa propre langue, il a sa singularité, sa muse pour bâtir et construire.La graduation de l'acte de la composition d'une œuvre est comparée à la construction d'un bâtiment.

Il donne plusieurs images à cet acte de l'écriture et porte à la fois un effet appréciatif et dépréciatif : «Un morceau de sa viande dans les bras. Cette nourriture qui nous mange. Et les voitures qui ne s'arrêtent pas. Qui en voudrait de cette viande morte. Chacun en a déjà bien assez de sa propre charogne»(N.C.B.A.N.Y, p.96). Il définit la muse de chacun comme un «petit manteau de douleur»; un " tricot de peau humide et piquant autour de l'âme», un «vertige à l'intérieur de lui-même, son propre gouffre ».

Mais comment les mots naissent-ils dans ce gouffre? Comme la pluie, ils viennent gratuitement en laissant leurs échos. Ceci nous rappelle la description de la composition surréaliste indiquée dans le Manifeste du surréalisme d'André Breton ${ }^{(1)}$. T.Vinausitue sa longue plaine dans le Luberon où «la lumière également est volubile ».Une plaine qui comprend aussi plusieurs endroits et des êtres. Il y décrit ses visites et se souvient d'une adolescente qui

1 BRETON André, Manifeste su surréalisme 1924, https://poesiemuziketc.wordpress.com/2013/03/29/andre-breton-manifeste-dusurrealisme/ consulté le 6 mai 2016 consulté le 6 mai 2016. 
écrit des poèmes. Dans cette longue plaine les livres sont comparés à la lune, aux magiciens, aux lettres « que l'on plante comme des arbres. Et qui poussent dans le cœur des gens ».

Puisqu'il s'assimile à une fenêtre, la lecture des dernières pages ressemble aux volets fermés. Il situe même son lecteur en lisant ses dernières pageset en lui créant plusieurs images : « Après le virage, on jette un dernier coup d'œil, au fond », «Les volets sont fermés», «La tonnelle désertée », « Le carré du cimetière », «Le rond du chaudron», «Les accès sont juste dégagés », «Il y a du sirop, renversé dans les herbes », «Les fourmis qui arrivent »(N.C.B.A.N.Y, p.100).

Sur le plan de la réception littéraire,Nos cheveux blanchiront avec nos yeuxfigure parmi les nouveautés littéraires présentées à la bibliothèque municipale de $\mathrm{P}$. Reverdy et Stéphane Aubron propose le café littéraire comme un «ovni $»^{(1)}$. Dans son article intitulé Dans la peau d'un livre, Catherine Simon fait parler le roman: « Je fais partie des quelques 660 ouvrages littéraires publiés pour la rentrée de septembre. Avalanche monstrueuse $»^{(2)}$.

1 LOIR Sarthe, Les nouveautés littéraires présentées autour d'un café ou d'un thé à la bibliothèque municipale $\mathrm{P}$. Reverdy, 17 novembre 11, Le Maine libre, dossier de presse envoyé d'Alma.

2 SIMON Catherine, Dans la peau d'un livre,Le Monde des livres, 19 août 2011, dossier de presse envoyé d'Alma. 
Le livre estégalement présenté dans la librairie le Bleuet à Banon, où l'on fait confiance aux petits éditeurs ${ }^{(1)}$. Par ailleurs, T. Vinau est invité dans les cafés littéraires, «il s'installe avec vigueur dans le paysage littéraire français, contrairement à $1 \%$ des écrivains de l'Hexagone» ${ }^{(2)}$.

Confrontée à cette année électorale 2012 «dense et passionnée qui a pour effet de "vitrifier" le marché», la nouvelle et toute jeune maison d'édition $\mathrm{Alma}^{(3)}$ qui a publié Nos cheveux blanchiront avec nos yeux, «étrennela rentrée littéraire $)^{(4)}$ avec l'une des synergies et titre à fort potentiel commercial $^{(5)}$. Présenté dans le dossier de la rentrée littéraire 2011dans Livres Hebdo du premier juillet, et l'année suivante dans Livres de France, il figure comme l'un des meilleurs soixante-quatorze romans présentés et encadrés.

1 FIGAROLI Roberto, La Provence, 4 septembre 2011, dossier de presse envoyé d'Alma.

2 L'écrivain vauclusien secoue la rentrée littéraire,La Provence, 20 octobre 212, dossier de presse envoyé par Alma.

3 BEUVE-MÉRY Alain, A quitte ou double pour les éditeurs, Le Monde.fr, 7 juillet 2011, dossier de presse envoyé d'Alma.

4 DELABROCHE Philippe, Lire, septembre 2011, dossier de presse envoyé d'Alma.

5 Livre hebdo Newsletter, 20 août 2012, dossier de presse envoyé d'Alma. 


\section{Conclusion}

Avec le roman poétique, le passé et l'avenir du roman se rencontrent au XXIe siècle. Un siècle qui témoigne de l'indétermination générique ou composite. La poésie dépasse les mots et les paroles, sa conception s'épanouit. Avec son roman poétique monologique Nos chevaux blanchiront avec nos yeux, la richesse des procédés stylistiques utilisés : la sonorité des mots, la tonalité poétique, l'écriture impressionnante, serrée, les richesses sémiotiques de la description poétique des paysages, des personnages, le jeu des miroirs des mots et des images, le texte peut laisser des empreintes profondes chez le lecteur. La prose flamboyante par le titrage, le desinit, laclausule, les thèmes du voyage, de l'enfance, de l'espoir représentent le point culminant de l'intrigue. L'amplification du thème du voyage accorde au roman une sorte de diversité par le déplacement du cosmopolite Walther.

Avec la fonction mimésique de la description, la structuration espace- temps peut être considérée comme la pierre de touche du roman poétique. L'absence de dialogue entre Walther et Sarah captive le lecteur pour découvrir deux modes de dénouement : coupure par le départ de Walther qui représente l'acmé de l'action et conversion par son retour. 
Avec le roman poétique, le lecteur peut-il se poser la question : où est-il question de savoir où l'on se sent le mieux ? Qu'est-ce que j'en fais-moi de tout ça ?

Serez-vous parmi ces lecteurs qui font « respirer (sa) forêt », « pousser (ses) troncs tordus » et «(ses) herbes bancales ».

Serez-vous parmi ceux « qui donnent du souffle à (ses) pétales et du jus à (ses) épines »?

Un roman tel que celui de Vinau est peut-être la preuve que l'expression poétique a encore beaucoup d'avenir... 


\section{BIBLIOGRAPHIE SELECTIVE}

\section{CORPUS}

VINAU Thomas, Nos cheveux blanchiront avec nos yeux, $2011^{1}$

\section{AUTRES QEUVRES DE THOMAS VINAU}

- Bric à BracHopperien, 2012

- Ici ça va, 2012

- La part des nuages, 2014

\section{OUVRAGES GÉNÉRAUX}

-ADAM Jean-Michel et PETITJEAN André, Le Texte descriptif:

\section{Poétique}

historique et linguistique textuelle, Paris, Armand Colin, 2006.

- ASTRE Marie-Louise et COLMEZ Françoise, Poésie française : anthologie

critique, Paris, Bordas, 1982.

- BÉNAC Henri et RÉAUTÉ Brigitte, Vocabulaire des études littéraires,

Paris, Hachette, 1993.

- BOUQUET Dominique, Le Surréalisme en France et en Europe, Collection les guides Pocket Classique Paris, Pocket, 2003. -CAILLAUD-ROBOAM Laurence, Les Figures de style illustrées par des

dessins de Plantu, Paris, Hatier, 2011.

-JAKPSON Roman, Huit questions de poétique, Paris, Seuil, 1997. -JOUVE Vincent, La Poétique du roman, Paris, Armand Colin, 2001. -RAIMOND Michel, La Crise du roman des lendemains des naturalistes aux années vingt, Paris, José Corti,1966.

1 Sauf indication, toute l'œuvre de Thomas Vinau est éditée à Paris chez Alma.

$-37-$ 
- SAYED Fathi, La Syntaxe et l'effectivité, Le Caire, Les amis du livre, 2000.

-VALETTE Bernard, Le Roman : initiation aux méthodes et aux techniques

d'analyse littéraire, Paris, Armand Colin, 2011.

\section{ARTICLES ET DOSSIER DE PRESSE ENVOYÉ D'ALMA}

-BARLAND J-R., « Thomas Vinau : homme cherchant refuge »,La provence, 19

octobre2014.

- BERLAND Daniel, « Fragments d'un immobile voyage »,Page, octobre 2011.

- BEUVE-MÉRY Alain, A quitte ou double pour les éditeurs, Le Monde.fr, 7

juillet 2011.

-COMBE Dominique, « Lire la poésie, lire le roman, selon Valéry : une

phénoménologie de la lecture », Littérature, n59, 1985, pp.57-70.

-DENIS Benoît, « Le roman poétique en 1929 », Textyles, nº 21, 2002, 31-40.

-DELABROCHE Philippe, « Lire », septembre 2011.

- DUFRAISSE Anthony, «InVinauveritas »,Le Matricule des anges, février 2014,

-FIGAROLI Roberto, «La Provence », 4 septembre 2011.

- GARCIA Cathy, « Juste après la pluie, Thomas Vinau », La cause littéraire, 12 juin 2014.

-GRANDMANGIN Rachel, « Le roman en vers »,Le magazine littéraire, $\mathrm{n}^{\circ} 477$,

Juillet-août 2008, pp.18-19.

- HELISSEN Alain, Cahier critique de poésie, nº 23, 2012.

- LAHAIE Christiane, « Entre géographie et littérature ; question de la mimèsis "),

Cahier de géographie du Québec, vol.52, nº147, 2008, p.439-451.

- LOIR Sarthe, «Les nouveautés littéraires présentées autour d'un café ou d'un 
thé à la bibliothèque municipale $\mathrm{P}$. Reverdy », Le Maine libre, 17 novembre 11 .

- LORET Éric et BOURMEAU Sylvain, « Ces images correspondent à mon

Travail », Libération, 6/7 octobre 2012.

- PLOUGASTEL Yanne, Le Monde Magazine, 10 sept 2011.

- R. V., Entre chien et loup, Livres Hebdo, n 869, vendredi 10 juin 2011.

-ZAMARI Philippe, « La BD en flagrant délire de Desproges-mania », Nice

Matin, 27 novembre 2011.

- CfL 'Yonne Républicaine, 24 novembre 2014.

-Livres Hebdo, 1 juillet 2011.

-Livre de France, septembre 2011.

-Libération, 22sept 2011.

-La dépêche, 14 septembre 2011.

- Livre hebdo Newsletter, 20 août 2012.

-La bibliothèque Méjanes fête ses 213 ans aujourd'hui, La provence, 19 novembre 13.

-Décapage, printemps-été 13.

\section{DICTIONNAIRES ET ENCYCLOPÉDIES}

- DE FOVILLE Jean-Marc, Les Pronoms de vos enfants : origine, histoire, signification, Paris, Hachette 1995.

- RICALENS-POURCHOT Nicole, Dictionnaire des figures de style, Paris,

Armand Colin, 2011.

-ROBERT Paul, Petit Robert, Paris, éd. Le Robert, 1991.

\section{SITOGRAPHIE}

- BRETON André, Manifeste su surréalisme 1924,https://poesiemuziketc. wordpress.com/2013/03/29/andrebreton-manifeste-du-surrealisme/ consulté le 6 mai 2016 consulté le 6 mai 2016. 
- VANEL Hervé, Manifeste du surréalimse (A. Breton), EncyclopædiaUniversalis [en ligne], consulté le 6 mai 2016. URL : http://www.universalis.fr/encyclopedie/manifeste-du-surrealisme/ -http://etc-iste.blogspot.com.eg/sité consulté le 19 février 2106 -http ://www.lefigaro.fr.site consulté le 18 février 2016

- https://twitter.com/thomasvinau

-www.vogue.fr

-http://www.cnrtl.fr/lexicographie/roman

ñ $\{$ Ó 